\title{
Case Report: Angiostrongylus cantonensis Meningoencephalitis in a 9-Month-Old Baby in Vietnam
}

\author{
Hien Pham Thu, ${ }^{1 *}$ Nam Đao Huu, ${ }^{2}$ Trang Le Thi Thu, ${ }^{3}$ and Lam Nguyen Van ${ }^{4}$ \\ ${ }^{1}$ International Outpatient Department of Vietnam National Children's Hospital (VNCH), Hanoi, Vietnam; ${ }^{2}$ Clinical Medicine of Children Infectious \\ Diseases Centre of Vietnam National Children's Hospital (VNCH), Hanoi, Vietnam; ${ }^{3}$ International Department of Vietnam National Children s' Hospital \\ (VNCH), Hanoi, Vietnam; ${ }^{4}$ Clinical Medicine of Children Infectious Diseases Centre of Vietnam National Children's Hospital (VNCH), Hanoi, Vietnam
}

\begin{abstract}
Meningoencephalitis is not a rare disease in small children. However, eosinophilic meningitis due to Angiostrongylus cantonensis is unusual in a baby. We describe the case of a 9-month-old baby from North Vietnam with eosinophilic meningoencephalitis. The baby lived in a rural area, where farming is widespread, and presented with fever and seizures. Laboratory results showed peripheral eosinophilia (16.1\%), cerebrospinal fluid (CSF) white blood cell count $220 / \mathrm{mm}^{3}$ (26\% eosinophils), CSF antibody test positive for Ascaris, CSF ELISA positive for Angiostrongylus cantonensis, and blood ELISA positive for $A$. cantonensis. A mobile worm was identified in the CSF. The presentation was consistent with a diagnosis of $A$. cantonensis eosinophilic meningitis. The baby recovered fully after administering albendazole (200 $\mathrm{mg} /$ day for 2 weeks), and intravenous dexamethasone $(0.6 \mathrm{mg} / \mathrm{kg} / \mathrm{day}$ every 8 hours) and mannitol (1.5 g/kg/day every 8 hours) for the first 3 days, followed by 5 days of oral prednisolone ( $2 \mathrm{mg} / \mathrm{kg} /$ day).
\end{abstract}

\section{INTRODUCTION}

Angiostrongylus cantonensis, also called the rat lungworm, can infect humans, causing meningitis. After ingestion by humans, the third-stage larvae migrate to the nervous system and molt to stage 4 and stage 5 young adult worms. ${ }^{1,2}$ Most of them die in the central nervous system (CNS) and, therefore, may not reach the pulmonary tract. ${ }^{3,4}$ The human immune response is activated because of the worms invading, living, and dying in the CNS.

Central nervous system invasion by $A$. cantonensis can cause many clinical manifestations. Most of the patients have meningitis with seizures, headache, stiff neck, and increase in eosinophil counts in the blood and spinal fluid. In addition, profound encephalitis can cause severe neurological symptoms, unconsciousness, and even death. Signs and symptoms of $A$. cantonensis-induced encephalitis and meningitis in children are not unique, except for a marked increase in eosinophils in both the blood and cerebrospinal fluid (CSF).

\section{CASE REPORT}

A 9-month-old boy presented to the Emergency and Poison Control Center in the Vietnam National Children's Hospital (VNCH) with chief complaints of fever and seizures. He had a 7day history of fever before presentation. The first seizure was witnessed by his parents at home the night before admission and lasted for 5 minutes. The child's head turned to the left side, the eyes rolled back, and there were convulsive movements with limb shaking, and he became unconscious. He was referred to the provincial hospital with suspected meningoencephalitis. However, the seizures continued, and, subsequently, he was transferred back to the $\mathrm{VNCH}$. The child did not have photophobia. There was no history of head injury or chronic disease. Respiratory and cardiac examinations were unremarkable. The neurologic examination was positive for signs of increased intracranial pressure. Although the child was alert, his neck was

\footnotetext{
* Address correspondence to Hien Pham Thu, International Outpatient Department, Vietnam National Children's Hospital (VNCH), 18/879 La Thanh Rd., Dongda District, Hanoi 100000, Vietnam. E-mails: hienkhth@yahoo.com or hienpt.tccb@nch.org.vn
}

quite stiff. Two seizures similar to the previous ones were observed. The baby was provided oxygen using a mask, and intravenous midazolam was administered. Heart rate, $\mathrm{spO}_{2}$, and electrocardiogram monitoring were initiated. Blood, feces, urine, and CSF were collected for laboratory tests, and a cranial computed tomography (CT) was performed. The laboratory findings are shown in Table 1, and an image of the worm in the CSF is shown in Figure 1. A video of the worm in the CSF is available online.

The head CT scans before treatment are shown in Figure 2, and no abnormal findings were observed. Investigations showed a peripheral blood total white blood cell (WBC) count of $21 \times 10 \mathrm{~g} / \mathrm{L}$ with $16 \%$ of eosinophils. Stool and urine analyses did not reveal any abnormalities. A white-colored live and mobile parasite was observed in the CSF. The leukocyte count was $220 / \mu \mathrm{L}$, with $28 \%$ eosinophils in the CSF. Protein and glucose concentrations were $0.48 \mathrm{mg} / \mathrm{dL}$ and $2.12 \mathrm{mg} / \mathrm{dL}$, respectively.

The findings of cortical injury were visible in both the cerebral hemispheres; axial T1W magnetic resonance imaging (MRI) shows exaggerated meningeal enhancement at both the cerebral hemispheres, suggesting meningoencephalitis (Figure 3). The visualization of a mobile worm and CSF eosinophilia strongly suggested parasitic meningoencephalitis. However, assays for other pathogens were performed. PCR of the CSF for enterovirus, herpes simplex virus, Streptococcus pneumoniae, and Haemophilus influenzae type $\mathrm{B}$, and bacterial culture of the CSF were all negative. ELISA of the blood and CSF were positive for Angiostrogylus cantonensis. The diagnosis was eosinophilic meningitis due to $A$. cantonensis. The child was treated with oral administration of albendazole (200 mg/day) for a 2-week treatment course, and intravenous dexamethasone $(0.6 \mathrm{mg} / \mathrm{kg} /$ day every 8 hours) for a 3-daytreatment and oral prednisolon ( $2 \mathrm{mg} / \mathrm{kg} /$ day) for a 5 -daytreatment course, along with intravenous infusion of mannitol $(1.5 \mathrm{~g} / \mathrm{kg} /$ day every 8 hours) for 3 days. In addition, maintenance anticonvulsant drugs were administered (midazolam and phenobarbital) to prevent seizures. He showed a complete recovery clinically with a decline in the eosinophilia after 12 days of treatment. The head MRI of the patient on the day of discharge is shown in Figure 3. The patient's MRI image suggests full recovery. 
TABLE 1

Laboratory test results

Infection markers (blood)

WBC

21.78

Neut, \%

40.7

36.6

16.1

EO, \%

CRP

Electrolytes (blood)

$\mathrm{Na}^{+}$

$\mathrm{K}^{+}$

Calci

Glucose

121

4.1

2.44

5.5

CSF analysis results (a white-colored live

parasite was observed in the CSF)

Cell counts on admission

Neut, \%

Lym, \%

EO, \%

Mono, \%

Total

Cell counts after 4 days

Neut, \%

Lym, \%

EO, \%

Mono, \%

Total'

Biochemistry on admission

Protein

Glucose

Chlorate

Pandy's test

Biochemistry after 4 days

Protein

Glucose

Chlorate

Biology

PCR Enterovirus

PCR HSV

PCR Streptococus pneumoniae

PCR HI type B

CSF culture

CSF antibody

Angiostrongylus cantonensis

Ascaris

Cysticercus cellulosae

Echinococcus

Schistosoma

Strongyloides stercoralis

Taenia solium

Toxocara

Blood antibody

Angiostrongylus cantonensis

Ascaris

Cysticercus cellulosae

Echinococcus

Schistosoma

Strongyloides stercoralis

Taenia solium

Toxocara

CSF ELISA

Angiostrongylus cantonensis

Gnathostoma spinigerum

Strongyloides stercoralis

Taenia solium/cysticercosis

Toxocara canis

Blood ELISA

Angiostrongylus cantonensis

Gnathostoma spinigerum

Strongyloides stercoralis

Taenia solium/cysticercosis

26

33

26

22

220

40

16

17

45

22

0.48

2.12

106.5

Negative

0.22

3.03

107.1

Negative

Negative

Negative

Negative

Negative

No chemicals

Positive*

Negative

Negative

Negative

Negative

Negative

Negative

No chemicals

Positive $†$

Negative

Negative

Negative

Negative

Negative

Negative

Positive $\neq$

Negative

Negative

Negative

Negative

Positiveł

Negative

Negative

Negative

Negative

$\mathrm{CRP}=\mathrm{C}$-reactive protein; $\mathrm{EO}=$ eosinophil; $\mathrm{HSV}=$ herpes simplex virus; $\mathrm{Ka}+=$ potassium; Lym = lymphocyte; Mono = monocyte; Na+ = sodium; Neut = neutrophil; WBC = white blood cell. * The positive results show the available of Ascaris antibody in CSF.

t The positive result show the available of Ascaris antibody in blood.

†The positive result show the available of Anglostrongylus cantonensis via enzyme-linked immunosorbent assay (ELISA) in CSF.

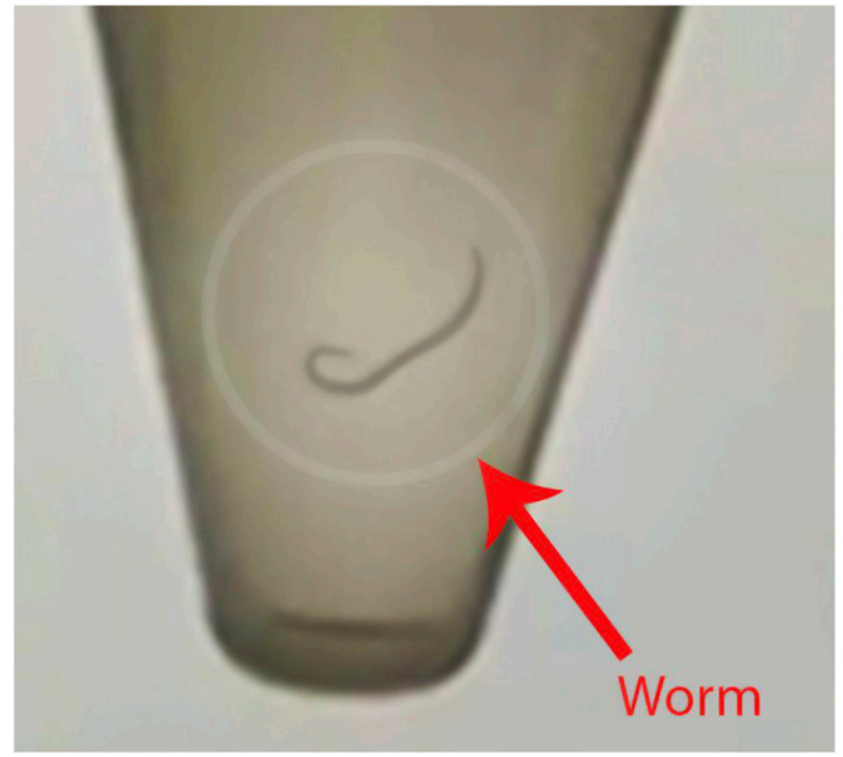

FIGURE 1. Photo of a worm in the cerebrospinal fluid (CSF). Supplemental Video. Video of a worm in the CSF. This figure appears in color at www.ajtmh.org.

\section{DISCUSSION}

Bacteria and viruses are more common than parasites as causes of meningoencephalitis in children. ${ }^{5}$ In this case, the high CSF WBC count suggested bacterial meningitis, but the relatively low neutrophil percentage suggested another diagnosis. However, the percentage of neutrophil was fairly small of $26 \%$ and $16 \%$, which were not suitable with the diagnosis of bacterial meningitis. The hallmark feature of Angiostrongylus eosinophilic meningitis (AEM) is the presence of eosinophilia, either in the CSF or in the peripheral blood. ${ }^{6}$ In this case, the patient was diagnosed with Angiostrogylus cantonensis. According to Nozais et al., ${ }^{7}$ hyper infection might develop through the dissemination of Strongyloides stercoralis in immunocompromised patients, particularly those under corticosteroid therapy. The patient in our case report was not immunocompromised, and there was no history of prolonged corticosteroid use.

Angiostrongylus cantonensis is found predominantly in Southeast Asia throughout the Pacific Basin, with cases reported in Thailand, Cambodia, Vietnam, Malaysia, India, Sri Lanka, Indonesia, the Philippines, Taiwan, China, Japan, Papua New Guinea, Hawaii, Tahiti, and several smaller Pacific islands. It is thought that infected rats (especially Rattus norvegicus) traveling in ships have spread the disease to Africa, Australia, New Zealand, Cuba, Puerto Rico, and Jamaica. Gnathostomiasis has a similar geographic distribution in Southeast Asia. ${ }^{8}$ Strongyloidiasis is an intestinal parasitosis that is frequently found in tropical and subtropical regions. ${ }^{7}$ Angiostrongylus cantonensis is the most common cause of eosinophilic meningitis in humans, which occurs after ingestion of raw or inadequately cooked intermediate hosts or food contaminated with infective third-stage larvae. Most of the cases present with subacute to chronic headache and fever, followed by acute coma. ${ }^{7}$ The characteristic symptoms are fever, severe headache, neck stiffness, and skin paresthesia. According to Wang et al.," in a study of 81 cases of 


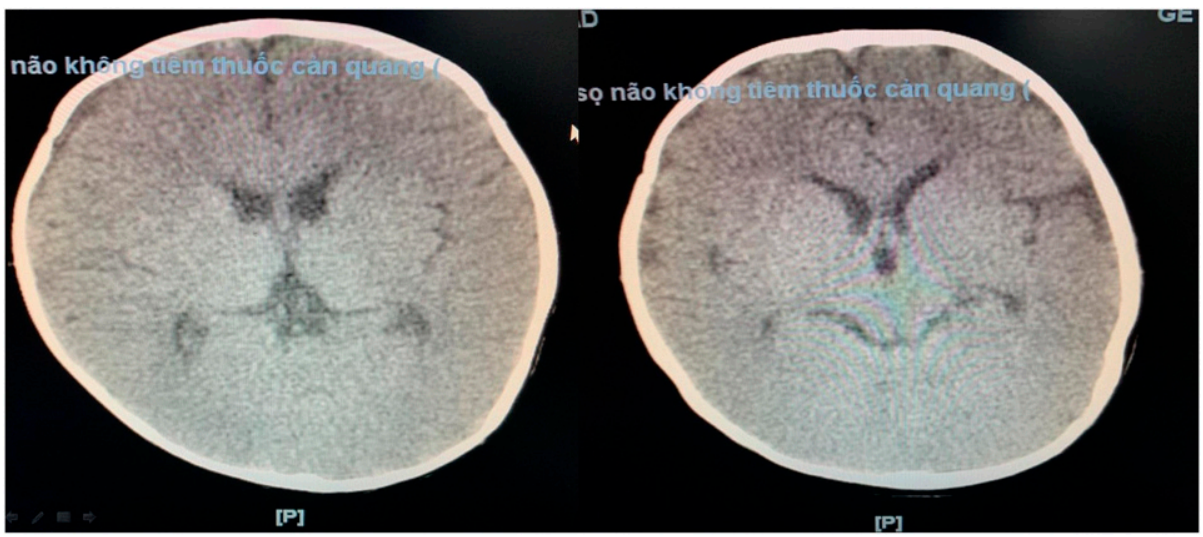

FiguRE 2. Computed tomography scans before treatment. No abnormal findings were observed. This figure appears in color at www.ajtmh.org.

A. cantonensis infection, peripheral eosinophilia was seen in $73 \%$ and CSF eosinophilia in $79 \%$. According to the CDC, risk factors for infection with $A$. cantonensis include the ingestion of raw or undercooked infected snails or slugs; or pieces of snails and slugs accidentally chopped up in vegetables, vegetable juices, or salads; or foods contaminated by the slime of infected snails or slugs. It is possible that ingestion of raw or undercooked transport hosts (freshwater shrimp, land crabs, frogs, etc.) can result in human infection, although this is less certain. In addition, contamination of the hands during the preparation of uncooked infected snails or slugs could lead to ingestion of the parasite. The current patient lived in a rural area in North Vietnam where agriculture is the main occupation. Because the child was crawling on the ground in an area infested with snails and rats, he might have been infected by the parasites or their eggs because of poor hygiene.

According to Wang et al., ${ }^{9}$ the average incubation period of A. cantonensis is 1-3 weeks but can range from 1 day to
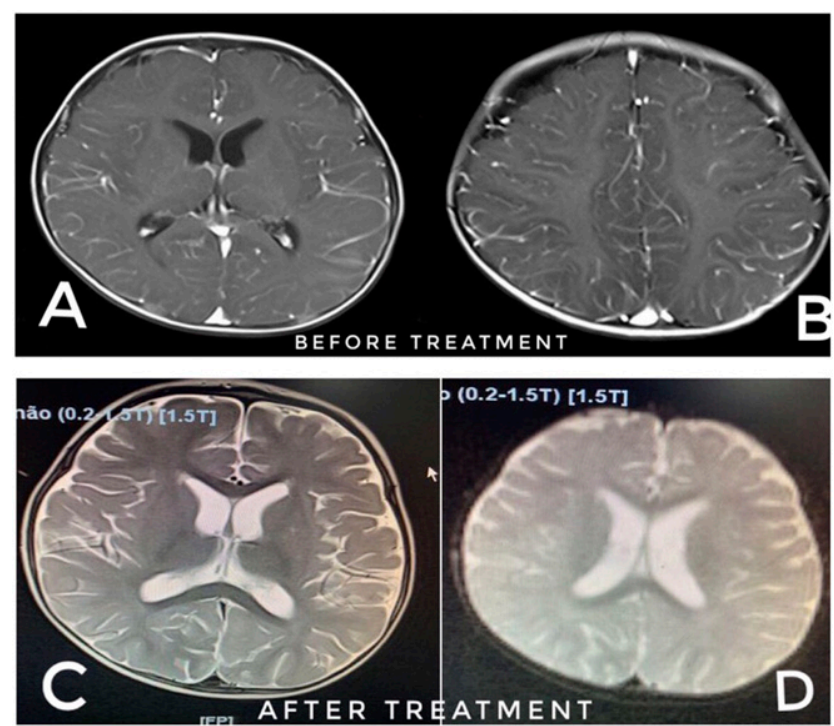

FIGURE 3. MRI before and after treatment. MRI images (A and B): MRI before treatment. The findings of cortical injury were visible in both the cerebral hemispheres, axial T1W MRI shows exaggerated meningeal enhancement at both cerebral hemispheres, suggesting meningoencephalitis. MRI image (C and $\mathbf{D})$ : MRI after treatment. The patient's MRI image after treatment suggests full recovery. This figure appears in color at www.ajtmh.org. greater than 6 weeks. The duration of the illness due to $A$. cantonensis is $2-8$ weeks but can last longer. Patients present with symptoms of bacterial meningitis, such as nausea, vomiting, neck stiffness, and headaches that are often global and severe. In addition, there might be complaints of abnormal sensations in the limbs. Sometimes, the eyes can be affected. $^{9}$ The main symptoms reported and their relative frequency are headache (100\%), photophobia or visual disturbance $(92 \%)$, neck stiffness $(83 \%)$, fatigue (83\%), hyperesthesia (75\%), vomiting (67\%), and paresthesia (50\%). Headaches were described as progressive and severe, and the cutaneous sensory findings were randomly located on the extremities and/or the trunk and did not have a specific dermatomal pattern of distribution. ${ }^{6}$ Our patient had seizures, fever, and increased intracranial pressure, but altered consciousness and paralysis were not seen.

The treatment for AEM and meningoencephalitis is not well defined and remains controversial. ${ }^{6}$ Murphy and Johnson suggested that albendazole should be administered for 2 weeks and that it is more effective when administered earlier in the course of illness. Prednisolone dose could be tapered after the 2-week course, if symptoms show an improvement, but should be administered for at least 2 weeks. ${ }^{6}$ The baby recovered fully after administering albendazole $(200 \mathrm{mg} / \mathrm{day}$ for 2 weeks), and intravenous dexamethasone $(0.6 \mathrm{mg} / \mathrm{kg} /$ day every 8 hours) and mannitol ( $1.5 \mathrm{~g} / \mathrm{kg} /$ day every 8 hours) for the first 3 days, followed by 5 days of oral prednisolone ( $2 \mathrm{mg} / \mathrm{kg} /$ day).

In conclusion, an increase in eosinophils in the blood and CSF is the key indicator of a parasitic infection. Hygiene, proper preparation of food, and handwashing are important measures for preventing these infections.

Received March 5, 2020. Accepted for publication April 28, 2020.

Published online May 18, 2020.

Note: Supplemental video appears at www.ajtmh.org.

Acknowledgments: We would like to thank Le Thanh Hai, director of the $\mathrm{VNCH}$, for providing general support, and the Center of Children Tropical Diseases of the $\mathrm{VNCH}$ for helping us in data collection. The American Society of Tropical Medicine and Hygiene (ASTMH) assisted with publication expenses.

Disclosure: The American Society of Tropical Medicine and Hygiene $(\mathrm{ASTMH})$ assisted with publication expenses.

Authors' addresses: Hien Pham Thu, International Outpatient Department of Vietnam National Children's Hospital (VNCH), Hanoi, 
Vietnam, E-mails: hienkhth@yahoo.com or hienpt.tccb@nch.org.vn. Nam Đao Huu, Clinical Medicine of Children Infectious Diseases Centre of Vietnam National Children's Hospital (VNCH), Hanoi, Vietnam, E-mail: namdhnt30@nch.org.vn. Trang Le Thi Thu, International Department of Vietnam National Children s' Hospital (VNCH), Hanoi, Vietnam, E-mail: lethutrang183@yahoo.com.vn. Lam Nguyen Van, Clinical Medicine of Children Infectious Diseases Centre of Vietnam National Children's Hospital (VNCH), Hanoi, Vietnam, E-mail: nguyenvanlam73@gmail.com.

\section{REFERENCES}

1. Mackerras MJ, Sandars D, 1955. The life history of the rat lungworm, Angiostrongylus cantonensis (Chen) (Nematoda: Metastrongylidae). Aust J Zool 3: 1-211.

2. Cowie RH, 2013. Biology, systematics, life cycle, and distribution of Angiostrongylus cantonensis, the cause of rat lungworm disease. Hawaii J Med Public Health 72: 6-9.

3. Tangchai P, Nye SWand Beaver PC, 1967. Eosinophilic meningoencephalitis caused by angiostrongyliasis in Thailand. Autopsy report. Am J Trop Med Hyg 16: 454-461.
4. Lindo JF, Escoffery CT, Reid B, Codrington G, Cunningham-Myrie C, Eberhard ML, 2004. Fatal autochthonous eosinophilic meningitis in a Jamaican child caused by Angiostrongylus cantonensis. Am J Trop Med Hyg 70: 425-428.

5. Behrman RE, Kliegman R, Jenson HB, eds. 2004. Nelson Textbook of Pediatrics, 17th edtion. Philadelphia, PA: Saunders, 2038-2047.

6. Murphy GS, Johnson S, 2013. Clinical aspects of eosinophilic meningitis and meningoencephalitis caused by Angiostrongylus cantonensis, the rat lungworm. Hawaii J Med Public Health 72: 35-40.

7. Nozais JP, Thellier M, Datry A, 2001. Disseminated strongyloidiasis. Presse Med 30: 813-818.

8. Lai $\mathrm{CH}$, Yen CM, Chin C, Chung HC, Kuo HC, Lin HH, 2007. Eosinophilic meningitis caused by Angiostrongylus cantonensis after ingestion of raw frogs. Am J Trop Med Hyg 76: 399-402.

9. Wang J, Qi H, Diao Z, Zheng X, Li X, Ma S, Ji A, Yin C, 2010. An outbreak of angiostrongyliasis cantonensis in Beijing. J Parasitol 96: 377-381.

10. Vand C, Sawanyawisuth K, 2002. Clinical manifestations and outcome of patients with severe eosinophilic meningoencephalitis presumably caused by Angiostrongylus cantonensis. Southeast Asian J Trop Med Public Health 33: 231-234. 